\section{D) Check for updates}

Cite this: Mol. BioSyst., 2017, 13, 1246

DOI: $10.1039 / \mathrm{c} 7 \mathrm{mb} 90021 \mathrm{j}$

rsc.li/molecular-biosystems

\title{
Correction: Metabolomic profiling and biochemical evaluation of the follicular fluid of endometriosis patients
}

\author{
Marianna Santonastaso, ${ }^{* a}$ Alessia Pucciarelli, ${ }^{b}$ Susan Costantini, ${ }^{c}$ Francesca Caprio, ${ }^{d}$ \\ Angela Sorice, ${ }^{C}$ Francesca Capone, ${ }^{c}$ Antonella Natella, ${ }^{d}$ Patrizia lardino, ${ }^{e}$ \\ Nicola Colacurci ${ }^{a}$ and Emilio Chiosi ${ }^{b}$
}

Correction for 'Metabolomic profiling and biochemical evaluation of the follicular fluid of endometriosis patients' by Marianna Santonastaso et al., Mol. BioSyst., 2017, DOI: 10.1039/c7mb00181a.

The author names were formatted incorrectly in the original article. The correct presentation of the author names is as given above.

The Royal Society of Chemistry apologises for these errors and any consequent inconvenience to authors and readers.

\footnotetext{
a Dipartimento della Donna, del Bambino e di Chirurgia Generale e Specialistica, Università degli Studi della Campania "Luigi Vanvitelli", Naples, Italy. E-mail: marianna.santonastaso@unicampania.it

${ }^{b}$ Dipartimento di Biochimica, Biofisica e Patologia Generale, Università degli Studi della Campania "Luigi Vanvitelli", Naples, Italy

"Unità di Farmacologia Sperimentale, Istituto Nazionale Tumori "Fondazione G. Pascale", IRCCS, Naples, Italy

${ }^{d}$ U.O.C. Ginecologia e Ostetricia - A.O.U. Università degli Studi della Campania "Luigi Vanvitelli", Naples, Italy

${ }^{e}$ U.O.C. Patologia Clinica e Molecolare - A.O.U. Università degli Studi della Campania "Luigi Vanvitelli", Naples, Italy
} 